Internist $2021 \cdot 62: 605-610$

https://doi.org/10.1007/s00108-021-01043-8

Angenommen: 22. April 2021

Online publiziert: 3. Mai 2021

(c) Der/die Autor(en) 2021

Redaktion

M. Hallek, Köln

Nach über 30 Jahren präklinischer

Entwicklung haben die CAR-T-Zellen (CAR chimärer Antigenrezeptor) mit der Zulassung der ersten CD19gerichteten Produkte nun auch Eingang in die klinische Routine gehalten. Da der Erkrankungsverlauf beim multiplen Myelom (MM) trotz beachtlicher therapeutischer Fortschritte weiterhin zumeist von wiederholten Rezidiven geprägt ist, liegt eine Erprobung von CART-Zellen zur Eliminierung maligner Plasmazellen auf der Hand.

\section{Status quo und therapeutische Herausforderungen beim multiplen Myelom}

Das MM, eine maligne Plasmazelldyskrasie, macht etwa $10 \%$ aller hämatologischen Neoplasien aus. Wenngleich die Erkrankung in vielen Fällen als unheilbar gilt, hat sich die Prognose für Betroffene in den letzten Jahren wesentlich verbessert. Entscheidend hierfür war der Einzug der monoklonalen Antikörper in die Standardtherapie. So erzielt die Induktionstherapie mit dem CD38-gerichteten Antikörper Daratumumab in Vierfachkombination mit Bortezomib, Dexamethason und Thalidomid bzw. Lenalidomid ein progressionsfreies Überleben („progression free survival“ [PFS]) von über $90 \%$ nach 18 [1] bzw. 24 Monaten [2].

Dennoch bleibt bei über einem Drittel der Patienten trotz optimalen Therapieansprechens eine minimale Resterkrankung („minimal residual disease“ [MRD]) nachweisbar, sodass Rezidive erwartbar sind und Folgetherapien weiterhin benötigt werden. Insbesondere für

\author{
X. Zhou $\cdot H$. Einsele $\cdot S$. Danhof \\ Medizinische Klinik und Poliklinik II, Universitätsklinikum Würzburg, Würzburg, Deutschland
}

\title{
CAR-T-Zell-Therapie beim multiplen Myelom
}

Patienten mit Hochrisikoerkrankung das heißt in Stadium III nach Revised International Staging System (R-ISS; [3]), mit extramedullärer Erkrankung („extramedullary disease“ [EMD]; [4]) oder ungünstigem Genexpressionsprofil [5] - sind daher neue Therapiealternativen erforderlich.

\section{》) Besonders Patienten mit multiplem Myelom und Hochrisi- kokonstellation benötigen neue Therapiealternativen}

Der erfolgreiche Einsatz von mit CAR modifizierten T-Zellen (CAR-T-Zellen) bei B-Zell-Neoplasien hat die Entwicklung von CAR-T-Zellen für die Behandlung anderer Krebserkrankungen derart beflügelt, dass für die Behandlung des MM gegenwärtig über 100 klinische Studien zur Untersuchung von CART-Zellen registriert sind (https://www. clinicaltrials.gov).

\section{Zielantigene für CAR-T-Zellen beim multiplen Myelom}

Das „B-cell maturation antigen“ (BCMA), auch bekanntals TNFRSF17 oder CD269, ist ein Membranprotein, das von reifen B-Zellen und sowohl gesunden als auch malignen Plasmazellen exprimiert wird. BCMA spielt eine signifikante Rolle bei der Proliferation der malignen Plasmazellen und kann dadurch den Erkrankungsprogress fördern. Auf der Zelloberfläche normaler hämatopoetischer Stammzellen oder gesunder Gewebe ist BCMA dagegen kaum oder nicht exprimiert. Deswegen stellt es ein sehr geeignetes Zielantigen für die CART-Zell-Therapie bei Patienten mit MM dar. Zurzeit ist die BCMA-gerichtete CAR-T-Zell-Therapie die am häufigsten untersuchte zelluläre Immuntherapie bei Patienten mit MM.

Prinzipiell sind andere Antigene mit Expression auf den malignen Plasmazellen ebenfalls mögliche Zielstrukturen für die CAR-T-Zell-Therapie beim MM. Präklinische und klinische Daten zeigten eine beachtliche Wirksamkeit von CAR-TZellen gegen CD138 (Syndecan-1; [6]),

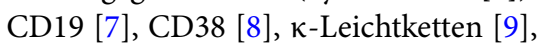
SLAMF7 (auch bekannt als CS1 oder CD319; [10]), GPRC5D [11], CD44v6 [12] und NKG2D [13]. CAR-T-Zell-Therapien gegen sämtliche der oben genannten alternativen Antigene werden derzeit im Rahmen klinischer Studien weiter evaluiert.

\section{Klinische Studiendaten}

Die aktuell verfügbaren Daten für den Einsatz von CAR-T-Zellen zur Behandlung des MM stammen in erster Linie aus klinischen Studien, die BCMA-gerichtete CAR-T-Zell-Therapien untersuchen [14]. So sind derzeit über 10 verschiedene BCMA-CAR-T-Zell-Produkte evaluiert bzw. publiziert. Insgesamt konnten BCMA-CAR-T-Zell-Therapien eine sehr gute Wirksamkeit mit einer hohen Gesamtansprechrate („overall response rate" $[\mathrm{ORR}])$ von bis zu $100 \%$ bei Patienten mit rezidiviertem/refraktärem $\mathrm{MM}$ erzielen [15]. Besonders erwähnenswert sind hierbei die Behandlungsergebnisse der KarMMa-Studie, in der Idecabtagen-Vicleucel (ide-cel, bb2121) in einem stark vorbehandelten Patientenkollektiv eine ORR von $73 \%$ und ein mittleres 
Tab. 1 Zusammenfassung der aktuell verfügbaren Studiendaten der CAR-T-Zell-Therapie bei rezidiviertem/refraktärem multiplem Myelom (publiziert als Manuskript oder Abstract)

\begin{tabular}{|c|c|c|c|c|c|c|c|c|}
\hline $\begin{array}{l}\text { Ziel- } \\
\text { antigen }\end{array}$ & Studie & Phase & $N$ & $\begin{array}{l}\text { Lymphozyten- } \\
\text { depletierende } \\
\text { Chemotherapie }\end{array}$ & Dosis (Zellen/kg) & $\begin{array}{l}\text { Anzahl der } \\
\text { Therapie- } \\
\text { linien }\end{array}$ & $\begin{array}{l}\text { Gesamt- } \\
\text { ansprechrate }\end{array}$ & PFS (Median) \\
\hline \multirow[t]{17}{*}{ BCMA } & \multirow[t]{2}{*}{ NCT02215967 } & 1 & 24 & $\mathrm{Cy} / \mathrm{Flu}$ & $0,3-9 \cdot 10^{6}$ & 9,5 & $81 \%^{\mathrm{a}}$ & 31 Wochen \\
\hline & & 1 & 12 & $\mathrm{Cy} / \mathrm{Flu}$ & $0,3-9 \cdot 10^{6}$ & 7 & $100 \%^{\mathrm{a}}$ & NA \\
\hline & NCT02546167 & 1 & 25 & Cy oder keine & $\begin{array}{l}\text { Kohorten } 1 \text { und 3: } \\
1-5 \cdot 10^{8} \\
\text { Kohorte } 2: 1-5 \cdot 10^{7}\end{array}$ & 7 & $\begin{array}{l}\text { Kohorte 1: } 44 \% \\
\text { Kohorte 2: } 20 \% \\
\text { Kohorte 3: } 64 \%\end{array}$ & $\begin{array}{l}\text { Kohorte 1: } 65 \text { Tage } \\
\text { Kohorte 2: } 57 \text { Tage } \\
\text { Kohorte 3: } 125 \text { Tage }\end{array}$ \\
\hline & NCT02658929 & 1 & 33 & $\mathrm{Cy} / \mathrm{Flu}$ & $\begin{array}{l}50,150,450 \text { oder } \\
800 \cdot 10^{6}\end{array}$ & $7-8$ & $85 \%$ & 11,8 Monate \\
\hline & \multirow[t]{2}{*}{ NCT03090659 } & 1 & 57 & Cy & $0,07-2,1 \cdot 10^{6}$ & 3 & $88 \%$ & 15 Monate \\
\hline & & 1 & 17 & Cy/Flu oder Cy & $0,21-1,52 \cdot 10^{6}$ & 4 & $88 \%$ & 12 Monate \\
\hline & \multirow[t]{2}{*}{ NCT03430011 } & $1 / 2$ & 19 & $\mathrm{Cy} / \mathrm{Flu}$ & $\begin{array}{l}50 \cdot 10^{6} \text { oder } \\
150 \cdot 10^{6}\end{array}$ & 10 & $100 \%$ & NA \\
\hline & & $1 / 2$ & 51 & $\mathrm{Cy} / \mathrm{Flu}$ & $\begin{array}{l}300,450 \text { oder } \\
600 \cdot 10^{6}\end{array}$ & 6 & $91 \%$ & NA \\
\hline & NCT03070327 & 1 & 11 & Cy/Flu oder Cy & $\begin{array}{l}72,137,475 \text { oder } \\
818 \cdot 10^{6}\end{array}$ & 6 & $64 \%$ & NA \\
\hline & NCT03338972 & 1 & 7 & $\mathrm{Cy} / \mathrm{Flu}$ & 5 oder $15 \cdot 10^{7}$ & 8 & $100 \%$ & NA \\
\hline & NCT03288493 & $1 / 2$ & 25 & $\mathrm{Cy} / \mathrm{Flu}$ & $0,5-5 \cdot 10^{8}$ & 7 & $48 \%$ & NA \\
\hline & NCT03274219 & 1 & 22 & $\mathrm{Cy} / \mathrm{Flu}$ & $\begin{array}{l}150,450,800 \text { oder } \\
1200 \cdot 10^{6}\end{array}$ & 7 & $83 \%$ & NA \\
\hline & NCT03548207 & $1 b / 2$ & 29 & $\mathrm{Cy} / \mathrm{Flu}$ & Median: $0,73 \cdot 10^{6}$ & 5 & $100 \%$ & NA \\
\hline & NCT03361748 & 2 & 128 & $\mathrm{Cy} / \mathrm{Flu}$ & $150-450 \cdot 10^{6}$ & 6 & $73 \%$ & 8,6 Monate \\
\hline & NCT03661554 & 1 & 16 & $\mathrm{Cy} / \mathrm{Flu}$ & $2-10 \cdot 10^{6}$ & NA & $100 \%$ & NA \\
\hline & NCT03093168 & 1 & 46 & $\mathrm{Cy} / \mathrm{Flu}$ & $9 \cdot 10^{6}$ & NA & $79,6 \%$ & 15 Monate \\
\hline & $\begin{array}{l}\text { NCT03716856 } \\
\text { NCT03302403 } \\
\text { NCT03380039 }\end{array}$ & 1 & 24 & $\mathrm{Cy} / \mathrm{Flu}$ & $\begin{array}{l}1,5,0,5,1 \text { oder } \\
1,8 \cdot 10^{8}\end{array}$ & 4,5 & $87,5 \%$ & NA \\
\hline CD138 & NCT01886976 & $1 / 2$ & 5 & PCD/CP/VAD & Median: $0,756 \cdot 10^{7}$ & 10 & $0 \%$ & NA \\
\hline CD19 & NCT02135406 & 1 & 10 & Mel + ASZT & $1-5 \cdot 10^{7}$ & 6 & $90 \%$ & 200 Tage \\
\hline NKG2D & NCT02203825 & 1 & 5 & Keine & $1 \cdot 10^{6}-3 \cdot 10^{7}$ & $\geq 5$ & $0 \%$ & NA \\
\hline $\begin{array}{l}\text { K-Leicht- } \\
\text { ketten }\end{array}$ & NCT00881920 & 1 & 7 & Cy oder keine & $0,92-1,9 \cdot 10^{8} / \mathrm{m}^{2}$ & 4 & $0 \%$ & NA \\
\hline $\begin{array}{l}\text { BCMA } \\
\text { und } \\
\text { CD19 }\end{array}$ & NCT03196414 & $1 / 2$ & 28 & $\mathrm{Cy} / \mathrm{Flu}$ & $\begin{array}{l}\text { BCMA: } 2-6,8 \cdot 10^{7} \\
\text { CD19: } 1 \cdot 10^{7}\end{array}$ & 3 & $92,6 \%$ & 8 Monate \\
\hline $\begin{array}{l}\text { BCMA } \\
\text { und } \\
\text { CD19b }\end{array}$ & NCT03455972 & $1 / 2$ & 32 & $\begin{array}{l}\text { Bu/Cy oder } \\
\text { Mel + ASZT }\end{array}$ & $\begin{array}{l}\text { CD19: } 1 \cdot 10^{7} \\
\text { BCMA: NA }\end{array}$ & NA & $100 \%$ & NA \\
\hline $\begin{array}{l}\text { BCMA } \\
\text { und } \\
\text { CD19 }\end{array}$ & $\begin{array}{l}\text { ChiCTR-OIC- } \\
17011272\end{array}$ & 2 & 22 & $\mathrm{Cy} / \mathrm{Flu}$ & $\begin{array}{l}\text { CD19: } 1 \cdot 10^{6} \\
\text { BCMA: } 1 \cdot 10^{6}\end{array}$ & 6 & $95 \%$ & $\begin{array}{l}\text { VGPR: } 243 \text { Tage } \\
\text { sCR: } 268 \text { Tage }\end{array}$ \\
\hline $\begin{array}{l}\text { BCMA/ } \\
\text { CD38 }\end{array}$ & ChiCTR1800018143 & 1 & 16 & $\mathrm{Cy} / \mathrm{Flu}$ & $\begin{array}{l}0,5,1,0,2,0,3,0 \text { oder } \\
4,0 \cdot 10^{6}\end{array}$ & NA & $87,5 \%$ & NA \\
\hline $\begin{array}{l}\text { BCMA/ } \\
\text { CD19' }\end{array}$ & NA & NA & 5 & $\mathrm{Cy} / \mathrm{Flu}$ & $1,0 \cdot 10^{6}$ oder $2,0 \cdot 10^{6}$ & 3 & $100 \%$ & NA \\
\hline $\begin{array}{l}\text { BCMA/ } \\
\text { TACI }^{\mathrm{C}}\end{array}$ & NCT03287804 & $1 / 2$ & 12 & $\mathrm{Cy} / \mathrm{Flu}$ & $\begin{array}{l}15,75,225,600 \text { oder } \\
900 \cdot 10^{6}\end{array}$ & 5 & $43 \%$ & NA \\
\hline \multicolumn{9}{|c|}{$\begin{array}{l}\text { ASZT autologe Stammzelltransplantation, BCMA } \\
\text { phosphamid, Flu Fludarabin, Mel Melphalan, NA } \\
\text { PFS „progression free survival” (progressionsfreie } \\
\text { cyclophilin ligand interactor", VAD Vincristin, Dox } \\
\text { 'Bei Patienten mit der höchsten CAR-T-ZellDosis } \\
\text { "Behandelt mit BCMA- und CD19-CAR-Zellen } \\
\text { 'Bi-spezifische CAR-T-Zellen }\end{array}$} \\
\hline
\end{tabular}


PFS von 8,8 Monaten erzielte. Ebenfalls für Aufsehen sorgte die CARTITUDE1-Studie, in der der Einsatz von LCARB38M im fortgeschrittenen Rezidiv zu einer ORR von $100 \%$ und einer PFS-Rate von $86 \%$ nach 9 Monaten führte. Die Daten einer aktualisierten Metaanalyse zeigten für 15 BCMA-CAR-T-Zell-Therapiestudien insgesamt eine hohe ORR von $82 \%$ mit einer Rate an Komplettremissionen („,complete remission“ [CR]) von $36 \%$ [16]. Auch bei Patienten mit Hochrisikomyelom und EMD wurde eine beachtlich hohe ORR von $78 \%$ berichtet, die mit sonstigen modernen Immunchemotherapien, beispielsweise Carfilzomib- oder Daratumumab-haltigen Regimen, nicht erreicht werden konnte [17, 18]. Diese vielversprechenden Ergebnisse aus klinischen Studien könnten zu einer baldigen Zulassung der BCMA-CAR-TZell-Therapie bei Patienten mit MM führen.

》 BCMA-CAR-T-Zell-Therapien
zeigten sehr gute Wirksamkeit
bei rezidiviertem/refraktärem
multiplem Myelom

Im Gegensatz zur BCMA-gerichteten CAR-T-Zell-Therapie ist die Entwicklung von Nicht-BCMA-CAR-T-Zellen noch weniger fortgeschritten. In klinischen Studien wurden weniger überzeugende Wirksamkeitsdaten für CD138-, NKG2D- und $\kappa$-Leichtketten-gerichtete CAR-T-Zell-Therapien berichtet $[9,13$, 19]. In einer Studie von Garfall et al. [20] wurde eine hohe ORR von 90\% nach CD19-gerichteter CAR-T-ZellTherapie bei Patienten mit rezidiviertem MM berichtet. Allerdings hatten diese Patienten eine zusätzliche Hochdosistherapie mit Melphalan und autologer Stammzelltransplantation vor der CART-Zell-Infusion erhalten [20]. Ergebnisse einer an unserem Zentrum initiierten Phase-I-Studie mit SLAMF7-gerichteten CAR-T-Zellen werden im Jahr 2021 erwartet (NCT04499339). Die klinischen Studiendaten ausgewählter CAR-T-ZellTherapien beim MM sind in - Tab. 1 zusammengefasst.

Internist 2021 · 62:605-610 https://doi.org/10.1007/s00108-021-01043-8

(c) Der/die Autor(en) 2021

X. Zhou $\cdot$ H. Einsele $\cdot S$. Danhof

CAR-T-Zell-Therapie beim multiplen Myelom

\section{Zusammenfassung}

Die Behandlung mit CAR-T-Zellen (CAR chimärer Antigenrezeptor) ist eine neuartige Strategie der zellulären Immuntherapie, die das patienteneigene Immunsystem als "Waffe gegen Tumorzellen" benutzt. Bei Patienten mit multiplem Myelom werden CAR-T-ZellTherapien im Rahmen klinischer Studien getestet. Die aktuellen Studiendaten der gegen das „B-cell maturation antigen“ (BCMA) gerichteten CAR-T-Zell-Therapien zeigen eine beachtliche Wirksamkeit, die eine baldige Zulassung erwarten lässt. Allerdings erleiden weiterhin die meisten Patienten nach einer Behandlung mit CAR-T-Zellen ein Rezidiv. Hinzu kommt, dass CAR-T-Zell-Therapien zu schwerwiegenden Nebenwirkungen wie Zytokinfreisetzungssyndrom und Neurotoxizität mit teilweise auch letalem Ausgang führen können. Ein angemessenes Kosten-Nutzen-Verhältnis der CAR-T-ZellTherapie stellt eine weitere Herausforderung dar. Trotz dieser Limitationen erscheint die CAR-T-Zell-Therapie eine attraktive Option für Patienten mit Myelom, sodass diese Therapie das Potenzial hat, in die Standardbehandlung integriert zu werden.

\section{Schlüsselwörter}

Adoptive Immuntherapie · T-Lymphozyten . Chimäre Antigenrezeptoren · „B-cell maturation antigen" . Unerwünschte Wirkungen der CAR-T-Zell-Therapie

\section{CAR T-cell therapy for multiple myeloma}

\begin{abstract}
Chimeric antigen receptor (CAR) T-cell treatment is a novel immunotherapy utilizing the patient's own immune system as a "weapon against tumor cells". In patients with multiple myeloma (MM), CAR T-cell therapy has been investigated in clinical trials. The current data on B-cell maturation antigen (BCMA)targeted CAR T-cells have shown impressive efficacy, and official approval is expected shortly. However, the majority of patients relapse after CAR T-cell therapy. Moreover, the treatment can cause severe adverse events such as cytokine release syndrome
\end{abstract}

and neurotoxicity with lethal outcome. The cost-benefit ratio of this treatment also needs to be optimized. Despite these limitations, CAR T-cell therapy represents an attractive option for patients with MM and has the potential to be incorporated into the standard of care.

\section{Keywords}

Immunotherapy, adoptive - T-lymphocytes . Receptors, chimeric antigen · B-cell maturation antigen - CAR T-cell therapy/adverse events

\section{Nebenwirkungen}

Im Vergleich zur konventionellen Immunchemotherapie hat die neuartige CAR-T-Zell-Therapie ein anderes $\mathrm{Ne}$ benwirkungsprofil, sogar Todesfälle wurden in verschiedenen Studien berichtet. Nach der CAR-T-Zell-Infusion zeigt die Mehrzahl der Patient eine Panzytopenie, welche die häufigste hämatologische Nebenwirkung ist. Die häufigsten nichthämatologischen Nebenwirkungen bei CAR-T-Zell-Therapie sind Zytokinfreisetzungssyndrom („cytokine release syndrome" [CRS]) und Neurotoxizität („immune effector cell-associated neurotoxicity syndrome“ [ICANS]).
Die Pathophysiologie von CRS und ICANS ist noch nicht vollständig geklärt. Ein möglicher Pathomechanismus ist eine überschießende Aktivierung der CAR-T-Zellen und anderer Immunzellen, was $\mathrm{zu}$ einer massiven Zytokinproduktion führen und infolgedessen Endothelschäden und Störungen der Blut-Hirn-Schranke verursachen kann. Das CRS kann sich in unterschiedlicher Ausprägung mit Fieber, Hypotonie und Hypoxie manifestieren, beim ICANS können Bewusstseinsstörung, Krampfanfälle, motorische Störungen oder sogar Hirnödeme auftreten. Auch wenn es bislang noch keine international einheitlichen Standards für die Behandlung von CRS und ICANS gibt, 


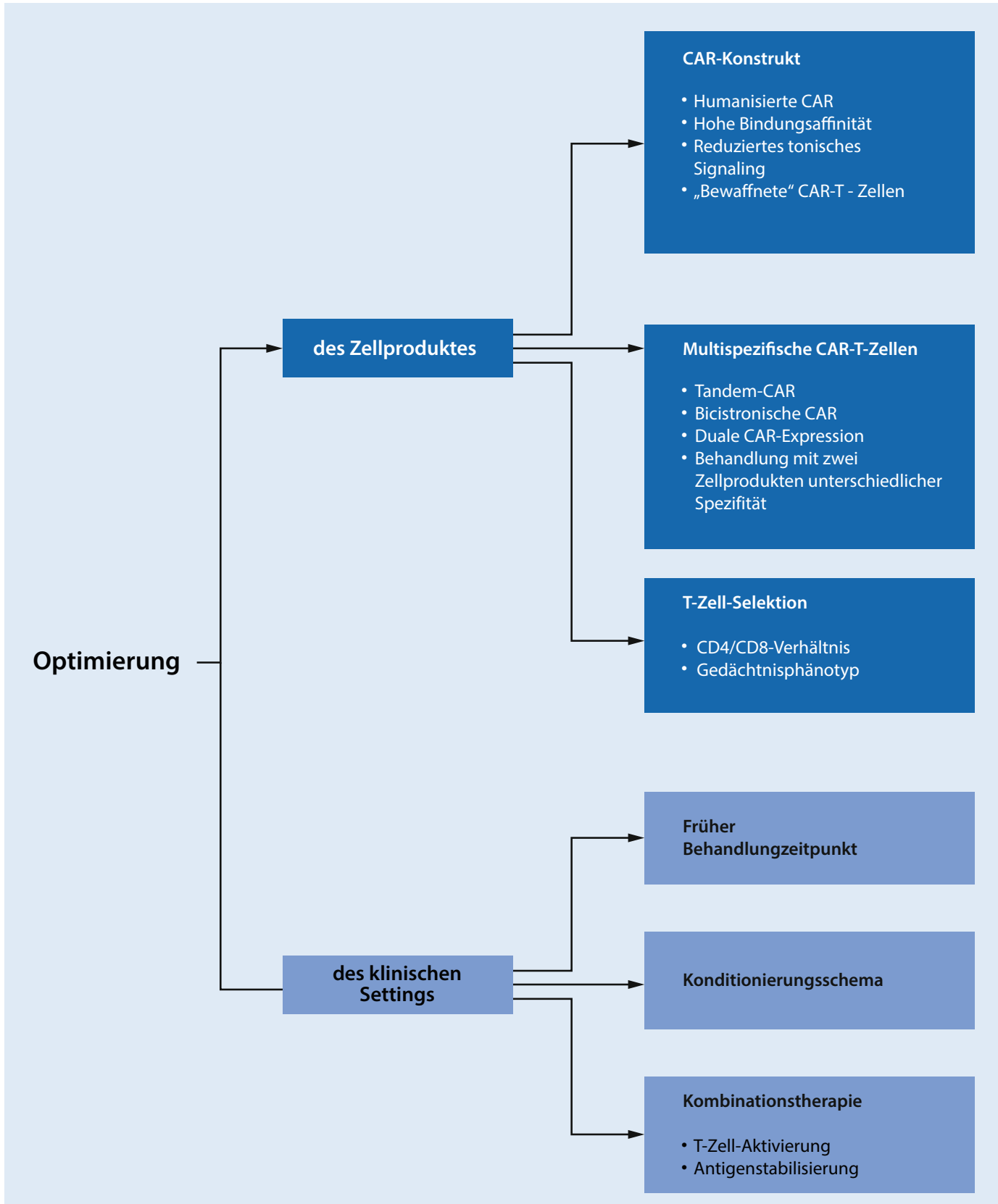

Abb. $1<$ Strategien zur Verbesserung der Effizienz der CAR-T-Zellen. CAR chimärer Antigenrezeptor

hat der Einsatz des Interleukin-6-Rezeptor-Blockers Tocilizumab beim CRS und von Glukokortikoiden beim ICANS einen gewissen Stellenwert erlangt.

\section{Resistenzmechanismen und neue Engineering-Strategien}

Trotz beeindruckender Erfolge erleiden die meisten Patienten mit Myelom nach einer Behandlung mit CAR-T-Zellen ein Rezidiv. So zeigte die bereits angesprochene Metaanalyse von 15 klinischen Studien mit Patienten, die im Rezidiv mit BCMA-CAR-T-Zellen behandelt wurden [16], zwar eine beeindruckende ORR (82\%) und CR-Rate (36\%), gleichzeitig wurden aber eine Rückfallrate von $45 \%$ und ein mittleres PFS von nur 10 Monaten dokumentiert. Ein derartiges Therapieversagen kann durch T-Zellen oder Tumorzellen vermittelt sein. Während dem T-Zell-vermittelten Therapieversagen häufig eine unzureichende Proliferation oder Persistenz der CAR-T-Zellen zugrunde liegt, steht beim tumorzellvermittelten Therapieversagen häufig der Verlust des Zielantigens im Vordergrund.

Strategien, um die Effizienz der CART-Zellen weiter $\mathrm{zu}$ steigern, sind vielfältig (•Abb. 1). Die präklinische Entwicklung fokussiert dabei auf die Konstruktion (1) voll humanisierter CAR zur Reduktion der Gefahr einer Abstoßungsreaktion gegen die CAR-T-Zellen (z.B. MCARH171 [11]) mit (2) optimierter Bindungsaffinität an das Tumorantigen (z.B. LCAR-B38M [21]) und (3) reduzierter tonischer Signalübertragung (z. B. auf Centyrin $^{\text {тx }}$ [Aro Biotherapeutics, Phi- 
ladelphia, PA, USA] basierende CAR-TZell-Produkte [22]), da diese mit vorzeitiger Erschöpfung der CAR-Effektorfunktionen einhergeht. Darüber hinaus setzt sich zunehmend die Überzeugung durch, dass gezielt selektierte T-Zell-Subpopulationen im Hinblick auf CD4/CD8-Verhältnis und Gedächtnisphänotyp einen Einfluss auf den Erfolg der CAR-T-ZellTherapie haben [23].

\section{I) Multispezifische CAR-T- Zell-Produkte sollen einem Antigenverlust auf den Tumorzellen entgegenwirken}

Um der Gefahr eines Antigenverlusts auf den Tumorzellen entgegenzuwirken, werden sogenannte multispezifische CAR-T-Zell-Produkte mit Reaktivität gegen mehrere Antigene entwickelt. Ein Beispiel hierfür ist ein CAR, dessen Bindedomäne an den körpereigenen Rezeptor APRIL („,a proliferation-inducing ligand“) angelehnt ist, der nicht nur BCMA, sondern gleichzeitig ein weiteres Tumorantigen namens TACI (,transmembrane activator and calcium modulator and cyclophilin ligand interactor") bindet [24] und somit die Wahrscheinlichkeit des Verlusts der Zielantigene reduziert. Andere bispezifische CAR-T-Zell-Produkte erkennen neben BCMA die Antigene CD19, CD38 oder SLAMF7 und befinden sich gegenwärtig in klinischer Testung. Vorläufige Daten aus einer Phase-1-Studie von BCMA/CD38-bispezifischen CAR-TZellen zeigen eine sehr hohe ORR von $87,5 \%$, und alle Patienten mit EMD haben eine Remission (partielle Remission oder besser) erreicht [25].

Neben Strategien, die in erster Linie auf eine optimierte Konstruktion der CAR-T-Zellen abzielen, gewinnt die Exploration des idealen klinischen Settings für die Behandlung zunehmend an Bedeutung. Durch oft langjährige intensive chemotherapeutische Vorbehandlung scheint die Fitness der T-Zellen von Patienten mit (mehrfach) rezidivierter Erkrankung deutlich geringer $\mathrm{zu}$ sein als zu Erkrankungsbeginn. Um dieser Hypothese auf den Grund zu gehen, schließen klinische Studien zunehmend auch nicht (z. B. KarMMa-4-Studie) oder nur gering vorbehandelte Patienten (z. B. CARTITUDE-2-Studie) ein. Auch die Frage nach geeigneten Kombinationstherapeutika wird vermehrt adressiert, insbesondere mit Blick auf Medikamente, welche die Antigenexpression auf Tumorzellen stabilisieren (etwa $\gamma$-Sekretase-Inhibitoren, NCT03502577) oder gleichermaßen myelomdepletierende und T-Zell-aktivierende Effekte aufweisen (z.B. Lenalidomid, NCT03070327).

\section{Praktische Therapiedurch- führung und Therapiekosten}

Im Unterschied zu den B-Zell-Neoplasien hat bislang kein CAR-T-Zell-Produkt für die Behandlung des $\mathrm{MM}$ eine $\mathrm{Zu}$ lassung durch die US Food and Drug Administration (FDA) oder Europäische Arzneimittel-Agentur (EMA) erhalten. Der Einsatz erfolgt daher bis auf Weiteres nur im Rahmen klinischer Studien. Diese
Studien werden den Nachweis erbringen müssen, dass die einmalige Behandlung mit CAR-T-Zellen der kontinuierlichen Standardmyelomtherapie überlegen ist, insbesondere im langfristigen Verlauf. In Anbetracht der enormen Fortschritte der letzten Jahre in der konventionellen Myelomtherapie hängt damit die Messlatte für eine Zulassung der CAR-TZellen beim MM ausgesprochen hoch. Zudem ist für einen breiten Einsatz ein angemessenes Kosten-Nutzen-Verhältnis erforderlich, wobei die Prognosen einen ähnlichen Kostenrahmen wie bei den CD19-CAR-T-Zell-Produkten vorsehen (300.000-500.000 \$). Sollten die CAR-T-Zellen einen relevanten Anteil an Patienten vom MM heilen können, dürfte dies allerdings kein Hindernis darstellen.

\section{Fazit für die Praxis}

- Die CAR-T-Zell-Therapie (CAR chimärer Antigenrezeptor) ist eine
Hier steht eine Anzeige.

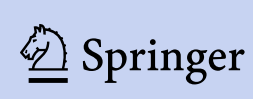


hochwirksame Behandlung für Patienten mit multiplem Myelom. Die baldige Zulassung eines ersten gegen das „B-cell maturation antigen“ (BCMA) gerichteten Zellprodukts wird erwartet.

- Aktuell sind in Deutschland CAR-TZell-Behandlungen von Patienten mit Myelom nur im Rahmen entsprechender Studien an geeigneten Zentren möglich.

- Die Verlängerung der Remissionsdauer ist aktuell die größte Herausforderung in der Entwicklung der CAR-T-Zell-Therapie beim MM.

- Für eine breite Anwendung ist ein akzeptables Kosten-Nutzen-Verhältnis der CAR-T-Zell-Therapie Voraussetzung.

\section{Korrespondenzadresse}

\section{Dr. med. X. Zhou}

Medizinische Klinik und Poliklinik II, Universitätsklinikum Würzburg Oberdürrbacher Straße 6, 97080 Würzburg, Deutschland zhou_x@ukw.de

\section{Einhaltung ethischer Richtlinien}

Interessenkonflikt. X.Zhou, H. Einsele und S. Danhof geben an, dass kein Interessenkonflikt besteht.

Für diesen Beitrag wurden von den Autoren keine Studien an Menschen oder Tieren durchgeführt. Für die aufgeführten Studien gelten die jeweils dort angegebenen ethischen Richtlinien.

Open Access. Dieser Artikel wird unter der Creative Commons Namensnennung 4.0 International Lizenz veröffentlicht, welche die Nutzung, Vervielfältigung, Bearbeitung, Verbreitung und Wiedergabe in jeglichem Medium und Format erlaubt, sofern Sie den/die ursprünglichen Autor(en) und die Quelle ordnungsgemäß nennen, einen Link zur Creative Commons Lizenz beifügen und angeben, ob Änderungen vorgenommen wurden.

Die in diesem Artikel enthaltenen Bilder und sonstiges Drittmaterial unterliegen ebenfalls der genannten Creative Commons Lizenz, sofern sich aus der Abbildungslegende nichts anderes ergibt. Sofern das betreffende Material nicht unter der genannten Creative Commons Lizenz steht und die betreffende Handlung nicht nach gesetzlichen Vorschriften erlaubt ist, ist für die oben aufgeführten Weiterverwendungen des Materials die Einwilligung des jeweiligen Rechteinhabers einzuholen.

Weitere Details zur Lizenz entnehmen Sie bitte der Lizenzinformation auf http://creativecommons.org/ licenses/by/4.0/deed.de.

\section{Literatur}

1. Moreau P et al (2019) Bortezomib, thalidomide and dexamethasone with or without daratumumab before and after autologous stem-cell transplantation for newly diagnosed multiple myeloma (CASSIOPEIA): a randomised, open-label, phase 3 study. Lancet 394(10192):29-38

2. Voorhees PM et al (2020) Daratumumab, lenalidomide, bortezomib, and dexamethasone for transplant-eligible newly diagnosed multiple myeloma: the GRIFFIN trial. Blood 136(8):936-945

3. Palumbo A et al (2015) Revised international staging system for multiple myeloma: a report from international myeloma working group. J Clin Oncol 33(26):2863-2869

4. Usmani SZ et al (2012) Extramedullary disease portends poor prognosis in multiple myeloma and is over-represented in high-risk disease even in the era of novel agents. Haematologica 97(11):1761-1767

5. Shah V et al (2020) Predicting ultrahigh risk multiple myeloma by molecular profiling: an analysis of newly diagnosed transplant eligible myeloma X trial patients. Leukemia 34(11):3091-3096

6. Sun C et al (2019) Safety and efficacy of targeting CD138 with a chimeric antigen receptor for the treatment of multiple myeloma. Oncotarget 10(24):2369-2383

7. Garfall AL et al (2015) Chimeric antigen receptor T cells against CD19 for multiple myeloma. $\mathrm{N}$ Engl J Med 373(11):1040-1047

8. Mihara K et al (2012) T-cell immunotherapy with a chimeric receptor against CD38 is effective in eliminating myeloma cells. Leukemia 26(2):365-367

9. Ramos CA et al (2016) Clinical responses with $\mathrm{T}$ lymphocytes targeting malignancy-associated kappa light chains. J Clin Invest 126(7):2588-2596

10. Gogishvili T et al (2017) SLAMF7-CAR T cells eliminate myeloma and confer selective fratricide of SLAMF7(+) normal lymphocytes. Blood 130(26):2838-2847

11. Smith EL et al (2018) Development and evaluation of an optimal human single-chain variable fragment-derived BCMA-targeted CAR T cell vector. Mol Ther 26(6):1447-1456

12. Carrabba MG et al (2018) Phase I-lla Clinical Trial to Assess Safety and Efficacy of MLMCAR44.1, a CD44v6 Directed CAR-T in Relapsed/ Refractory Acute Myeloid Leukemia (AML) and Multiple Myeloma (MM). Blood 132(Supplement 1):5790-5790

13. Baumeister SH et al (2019) Phase I trial of Autologous CAR T cells targeting NKG2D Ligands in patients with AML/MDS and multiple myeloma. Cancer Immunol Res 7(1):100-112

14. Moreau P et al (2019) Chimeric antigen receptor T-cell therapy for multiple myeloma: a consensus statement from The European Myeloma Network. Haematologica 104(12):2358-2360

15. Li C et al (2019) Clinical responses and pharmacokinetics of fully human BCMA targeting CAR T cell therapy in relapsed/refractory multiple myeloma. Clin Lymphoma Myeloma Leuk 19(10):e23-e24

16. Gagelmann N et al (2020) B cell maturation antigen-specific chimeric antigen receptor T cells for relapsed or refractory multiple myeloma: a meta-analysis. Eur J Haematol 104(4):318-327

17. Lonial S et al (2016) Daratumumab monotherapy in patients with treatment-refractory multiple myeloma (SIRIUS): an open-label, randomised, phase 2 trial. Lancet 387(10027):1551-1560
18. Zhou X et al (2020) Carfilzomib based treatment strategies in the management of relapsed/ refractory multiple myeloma with extramedullary disease. Cancers 12(4). https://doi.org/10.3390/ cancers12041035

19. Guo B et al (2016) CD138-directed adoptive immunotherapy of chimeric antigen receptor (CAR)-modified T cells for multiple myeloma. J Cell Immunother 2(1):28-35

20. Garfall AL et al (2018) Anti-CD19 CAR T cells with high-dose melphalan and autologous stem cell transplantation for refractory multiple myeloma. JCIInsight3(8). https://doi.org/10.1172/jci.insight. 120505

21. Zhao WH et al (2018) A phase 1, open-label study of LCAR-B38M, a chimeric antigen receptor T cell therapy directed against $B$ cell maturation antigen, in patients with relapsed or refractory multiple myeloma. JHematol Oncol 11(1):141

22. Costello CL et al (2019) Phase 2 study of the response and safety of P-Bcma-101 CAR-T cells in patients with relapsed/refractory $(r / r)$ multiple myeloma (MM) (PRIME). Blood 134(Supplement_1):3184-3184

23. Wang Metal (2019) Identification and validation of predictive biomarkers to CD19- and BCMA-specific CAR T-cell responses in CAR T-cell precursors. Blood 134(Supplement_1):622-622

24. Popat R et al (2019) Phase 1 first-in-human study of AUTO2, the first chimeric antigen receptor (CAR) T cell targeting APRIL for patients with relapsed/refractory multiple myeloma (RRMM). Blood 134(Supplement_1):3112-3112

25. Li C et al (2019) A Bispecific CAR-T cell therapy targeting Bcma and CD38 for relapsed/ refractory multiple myeloma: updated results from a phase 1 dose-climbing trial. Blood 134(Supplement_1):930-930 